\title{
Laser-Directed Self-Assembly of Block Copolymers Investigated with Synchrotron GISAXS
}

\author{
Pawel W. Majewski ${ }^{a}$, Kevin G. Yager ${ }^{b}$ \\ ${ }^{a}$ Department of Chemistry, University of Warsaw, Warsaw, Poland \\ ${ }^{b}$ Center for Functional Nanomaterials, Brookhaven National Laboratory, Upton, NY, USA \\ AuthorEmail:pmajewski@chem.uw.edu.pl
}

We utilize synchrotron GISAXS technique to characterize the process of laser-directed self-assembly of block copolymer (BCP) thin films. Our method, called Laser Zone Annealing (LZA) accelerates the otherwise slow kinetics of morphology development in BCP by several orders of magnitude compared to conventional thermostatic annealing. The acceleration results from extremely steep, but short-lasting temperature transients, induced in the material by rastering narrowly focused laser line over the light-absorbing substrate. GISAXS studies are used to assess grain size in the films by measuring breadth parameter of the scattering peaks originating from periodic $\mathrm{BCP}$ nanostructure. This allows comparison between grain growth kinetics in LZA and conventional oven annealing. LZA, when coupled to photo-thermal shearing (Soft-Shear LZA), leads to a global orientation of block copolymer domains. The degree of alignment of BCP domains can be assessed by GISXAS, both qualitatively - by observing increased number of reflexes on diffraction patterns, and qualitatively - by analyzing peak-widths and scattering evolution during the inplane rotation of the sample. We demonstrate monolithic alignment for a range of block-copolymer materials including PS- $b$-PMMA, PS- $b$-PEO, PS- $b$-P2VP, PS- $b$-PI and observe different responsiveness to the shearing rate, depending on the characteristic relaxation timescale of the particular material. We also investigate a pathway-dependence of ordering in block copolymer films under ultra-fast soft-shear LZA conditions, which we term "latent ordering". Our findings help to understand fundamental mechanisms of the BCP self-assembly and to engineer an optimal approach for processing of these materials. In short perspective, we plan to extend our methodology by in-situ studies of BCP directed self-assembly with elements of automated exploration of parameter space via machine learning algorithms. 